\title{
El Adulto Mayor en la actualidad y la Diabetes Mellitus como una enfermedad preocupante
}

The Elderly at present and Diabetes Mellitus as a worrying disease

Adriana Mederos González. ${ }^{1}$ \& Msc. Eugenio González Pérez. ${ }^{2}$

Recibido: 13-01-2019 / Revisado: 15-02-2019 / Aceptado: 17-03-2019 / Publicado: 04-04-2019

DOI: https://doi.org/10.33262/anatomiadigital.v2i2.1081

\section{Abstract.}

The aging of the population is a universal phenomenon, framed at the crossroads of the drop in the birth rate and the increase in life expectancy due to medical advances, especially in countries with a good standard of living. In 2000, the years of the world population rose to an average of 65 years. 1

Exercise is a basic body need. The human body is made to be used, otherwise it deteriorates; if the exercise is renounced, the organism works below its physical possibilities, therefore it is abandoned from life. 2

Diabetes Mellitus is a heterogeneous syndrome caused by genetic-environmental interaction, characterized by chronic hyperglycemia, a consequence of a deficit in

\section{Resumen.}

El envejecimiento de la población es un fenómeno universal, enmarcado en la encrucijada del descenso de la tasa de natalidad y el aumento de la esperanza de vida por los adelantos médicos, sobre todo en los países de buen nivel de vida. En el año 2000 se elevó los años de la población mundial a un promedio de 65 años. 1

El ejercicio es una necesidad corporal básica. El cuerpo humano está hecho para ser usado, de lo contrario se deteriora; si se renuncia al ejercicio el organismo funciona por debajo de sus posibilidades físicas por tanto se abandona de la vida.2

La Diabetes Mellitus es un síndrome heterogéneo originado por la Interacción genético-ambiental, caracterizado por una

${ }^{1}$ Ciencias Médicas Mayabeque, Mayabeque, Cuba, amederos@infomed.sld.cu 0002-7353-0146

2 Ciencias Médicas Mayabeque, Mayabeque, Cuba, egonzalez@infomed.sld.cu 0002-7353-0147

https://orcid.org/0000-

https://orcid.org/0000- 
the secretion or action of insulin, which causes acute and chronic complications.

Keywords: Elderly, age, aging, Diabetes Mellitus, health.
Hiperglucemia crónica, consecuencia de un déficit en la secreción o acción de la insulina, que origina complicaciones agudas y crónicas.

Palabras claves: Adulto mayor, edad, envejecimiento, Diabetes Mellitus, salud.

\section{Introducción.}

La prolongación de la vida y la capacidad de trabajo de la persona de edad avanzada, es uno de los problemas sociales más importantes en los que debe incidir el profesional de la Cultura Física en la lucha por la salud y la longevidad. El envejecimiento se asocia a una reducción de la capacidad aeróbica máxima y de la fuerza muscular, así como de la capacidad funcional en general, así se puede considerar que el ser humano evoluciona físicamente hasta los 20 años e involuciona claramente entre los 65-70 años. Un cuerpo que no se ejercita utiliza solo el $27 \%$ de la energía posible de que dispone, pero este bajo nivel de rendimiento puede incrementarse hasta $56 \%$ si se práctica regularmente ejercicios, este aumento de crecimiento orgánico podrá ser apreciado en todos los ámbitos de la vida. La edad modifica los caracteres fisicoquímicos del gel coloidal y puede alterar la alimentación y la oxigenación de las células, acelerando así el desgaste y la sensibilidad a la inestabilidad reconocida de las suspensiones coloidales. Hace años se admitía que el número de fibras colágenas aumentada con la edad para un peso dado de tejido, y así, sin darse uno cuenta, el tejido flexible del individuo joven se convertía en el tejido rígido del anciano. Sin embargo, ya se ha demostrado que hay varias clases de colágenos en libertad en el protoplasma, de las cuales dos tienen su origen en transformaciones preliminares en el interior de la célula conjuntiva.4, 5

La diabetes mellitus (DM) es un conjunto de trastornos metabólicos, que afecta a diferentes órganos y tejidos, dura toda la vida y se caracteriza por un aumento de los niveles de glucosa en la sangre: hiperglucemia. La causan varios trastornos, siendo el principal la baja producción de la hormona insulina, secretada por las células $\beta$ de los Islotes de Langerhans del páncreas endocrino, o por su inadecuado uso por parte del cuerpo, que repercutirá en el metabolismo de los hidratos de carbono, lípidos y proteínas. La diabetes mellitus y su comorbilidad constituyen actualmente la principal causa de preocupación en salud pública.6

\section{Objetivos:}

- Enfatizar en aspectos relacionados con el Adulto Mayor.

- Profundizar en la Diabetes Mellitus como una de las principales enfermedades de la actualidad. 


\section{Desarrollo}

La orientación al adulto mayor pretende que las personas usen y desarrollen sus propias capacidades y utilizando los conocimientos e información necesarios, tengan la posibilidad de enfrentar en mejor forma sus problemas personales, de interrelación con otros y con el mundo que los rodea. La orientación es una acción educativa en la que pretende que los orientados vivan un proceso de aprendizaje, tanto en lo que se refiere a usar y desarrollar sus propias capacidades como la integración de los conocimientos e informaciones que necesiten. El propósito de la acción orientadora no es dirigir la vida de las personas, ni resolverle sus problemas; por el contrario es que ellas lo puedan hacer de una manera más integrada, más independiente, y mejor organizada. El envejecimiento mundial se ha ido acelerando desde 1980 en que existían 550000000 de adultos mayores, con una perspectiva demográfica de 1250000000 en el año 2025. En la década del noventa la Organización de Naciones Unidas implementó el primero de octubre como Día Internacional del Adulto Mayor, con el fin de favorecer la toma de conciencia sobre el valor de la prolongación de la vida y la necesidad de crear sociedades cada vez más integradoras y justas para todas las personas adultas mayores.7

En los últimos años ha supuesto un gran escándalo social, la comunicación de casos de indignante maltrato a personas mayores, por parte de sus propios familiares o personas de convivencia. Algunos varones son explotados por su familia, y algunas mujeres han llegado a sufrir el llamado "Síndrome de la Abuela Esclava" por la que algunas familias, abusan de la confianza que le supone, para que trabaje para ellos, sin que ellos tengan consideración con ella.

En la mayoría de los casos, el maltrato pasa a ser psicológico y casi imperceptible.

Otro tipo de caso es que la familia o convivientes ya no los quieren porque se ve a la persona como un estorbo, por lo general esta idea se da por falta de dinero para mantener tanto a él como al resto de los familiares o convivientes del hogar. Los asilos de ancianos también son criticados duramente por acusaciones graves de maltratos físicos y psicológicos, como también a falta de atención y el poco cuidado que reciben.

La Tercera Edad constituye una etapa de la vida muy influenciada, más bien determinada por la opinión social, por la cultura donde se desenvuelve el anciano. Hasta hoy día la cultura, de una forma u otra, tiende mayoritariamente a estimular para la vejez el sentimiento de soledad, la segregación, limitaciones para la vida sexual y de pareja, y de la propia funcionalidad e integración social del anciano.8

Metas de salud para los años tardíos 
A nivel mundial, el segmento de la población que está experimentando un crecimiento más rápido es el más viejo. La proporción de centenarios es la que más rápido crece en la población, seguida del grupo de 80 a 99 años de edad. Con el tiempo, al eliminar las principales epidemias de enfermedades infecciosas, el número de ancianos comenzó a ascender. Hoy, gracias a los extraordinarios progresos de la ciencia médica, sobre todo en el campo de la farmacología, los ciudadanos de la tercera edad se han convertido en un grupo de gran importancia. Se prevé que en el siglo XXI, la creciente población de personas mayores de 85 años — los "viejos-viejos"-, con su enorme consumo de servicios médicos, crearán importantes crisis económicas, de recursos médicos y éticas tanto en los países desarrollados como en los que se encuentran en vías de desarrollo.

El campo de la gerontología se esfuerza en seguir el ritmo de esta transición demográfica.

Cuanto más anciano es un grupo de personas, mayor variedad muestran sus integrantes. De hecho, las variaciones del funcionamiento físico, mental y social son mayores entre los ancianos que en cualquier otro grupo de edad.

El deterioro funcional que acompaña al envejecimiento puede posponerse manteniendo una vida física, mental y social activa. Por tanto, el objetivo de los programas de promoción de la salud dirigidos a las personas de edad avanzada no consiste en prolongar la vida indefinidamente, sino, ante todo, en dar la mejor vida posible a los años que le quedan a cada persona.

Una forma de contribuir a disminuir el gran consumo de recursos de salud de la población anciana consiste en reducir en lo posible el período de morbilidad terminal. Para ello hay que mantener a las personas lo más activas posible y capaces de cuidarse a sí mismas casi hasta su muerte. Con ello, disminuirá la duración, aunque no necesariamente la intensidad, de la atención médica que necesitan.

Por otra parte, se reducirá evidentemente el sufrimiento y se combatirá la sensación de deterioro de los ancianos y de los familiares que los cuidan.

Dados los muchos problemas de los niños y jóvenes de hoy, la tarea de los abuelos -incluso como trabajo no remunerado - podría ser uno de los mayores regalos sociales y económicos que la persona puede dar a su comunidad. Se puede pensar en ejemplos adicionales. Los beneficios psicológicos, sociales y culturales que pueden proporcionar los residentes más ancianos de la comunidad apenas si han empezado a descubrirse. 9

\section{Principales problemas de salud a estas edades}

\section{Mortalidad}


La esperanza de vida es la edad hasta la que sobrevive el individuo medio (en términos de edad) de una cohorte de población. Puesto que casi todos los países económicamente desarrollados tienen esperanzas de vida al nacer superiores a 65 años, más de la mitad de todas las muertes se producen a los 65 años o después.

Así pues, las causas de muerte en este estrato de edad dominan el total de causas del país. Ello significa que es imprescindible estudiar las causas de muerte y discapacidad de cada etapa de la vida por separado a fin de orientar los esfuerzos de prevención en etapas anteriores del ciclo vital.

Por tanto, no sorprende que las tasas brutas de mortalidad de los países, que dependen en gran medida de las muertes de los ancianos, muestren que las enfermedades cardiovasculares, las enfermedades cerebrovasculares, las neoplasias malignas, las enfermedades obstructivas del pulmón y las neumonías son las principales causas de muerte en casi todas las regiones del mundo, tanto en los países económicamente evolucionados como en los que todavía se encuentran en las primeras etapas de su evolución económica.

La mortalidad se acelera a medida que transcurren los decenios de la vida. A partir de los 25 a 34 años de edad, las tasas de mortalidad por todas las causas se duplican con cada decenio sucesivo, tanto en los hombres como en las mujeres, en casi todos los países. En cada decenio, las tasas de mortalidad de los hombres (por 100000 habitantes) son siempre superiores a las de las mujeres.

El factor multiplicador por decenio es incluso superior para algunas de estas causas.

En casi todos los países, la mortalidad total por enfermedades cardiovasculares es de dos a tres veces mayor a los 65 años que a los 55 y aumenta de nuevo de cuatro a cinco veces a partir de los 75 años. Las tasas de accidentes cerebrovasculares muestran una aceleración similar a partir de los 55 años. El número total de cánceres se duplica entre los 55 y los 65 años y de nuevo después de los 65 . Todo ello significa que las tasas de mortalidad se aceleran entre 10 y 15 veces durante los tres o cuatro últimos decenios del ciclo vital.

Esta información sobre la aceleración de las tasas de mortalidad que acompañan el envejecimiento revela tres hechos importantes:

- El rápido incremento de la vulnerabilidad en los ancianos.

- Las ganancias sociales potenciales que producirían programas efectivos de prevención temprana. Estos programas podrían retrasar esta oleada de enfermedad y muerte hasta etapas posteriores en el ciclo vital. 
- El enorme incremento de los costos de atención médica y en hogares de ancianos para el gran número de ancianos que permanecen discapacitados y dependientes, a menudo durante años, antes de morir de su enfermedad.10

\section{Enfermedades sistémicas generales y discapacidades}

Muchos ancianos sufren largos años de discapacidad, sintiéndose mal y funcionando mal, antes de morir. Hoy sabemos que pueden tomarse muchas medidas para reducir los riesgos y la gravedad de los procesos discapacitantes, quizás no para todos, pero sin duda para muchos de ellos. Las causas principales de sufrimiento y de debilidad abarcan tanto a las causas principales de muerte como también a otros tipos de problemas de salud.

Hasta 1990, la población mundial mayor de 60 años era menor que la de los otros grupos de edad. El número de mujeres sobrepasaba al de los hombres por una razón de 123 a 100 y las diferencias en el peso relativo de las distintas causas de discapacidad en hombres y mujeres eran mucho menores que en las personas de edad madura. En todas las regiones del mundo, entre $80 \%$ y $95 \%$ de los años de discapacidad pueden atribuirse a enfermedades no transmisibles. En conjunto, el impacto de las enfermedades transmisibles, obstétricas, perinatales y nutricionales desciende desde $46 \%$ a la edad de $0-4$ años a $6 \%$ en las personas de 60 años o más. De igual modo, el impacto de todas las lesiones sobre la discapacidad baja desde $18 \%$ a los $0-4$ años hasta $2,5 \%$ a partir de los 60 años.

\section{Factores de riesgo de las causas de muerte y discapacidad}

- El consumo de cigarrillos es uno de los principales factores contribuyentes al desarrollo de la cardiopatía isquémica (ataques al corazón), la enfermedad cerebrovascular (accidente cerebrovascular), la enfermedad pulmonar obstructiva crónica, la bronquitis y la neumonía o ambas, y muchos cánceres. El consumo de tabaco incrementa la magnitud potencial de enfermedad y sufrimiento y determina el tipo de muerte. Solo hay una solución: dejar de fumar.

- Las dietas ricas en grasa elevan el riesgo de cáncer de colon y próstata y, quizás, de otros cánceres. También influyen de forma determinante en la aparición de la aterosclerosis, que se expresa en forma de ataques al corazón y trombosis cerebrales.

- La hipertensión arterial es la enfermedad que en mayor medida contribuye al accidente cerebrovascular (tanto hemorrágico como trombótico), facilita los infartos de miocardio y, cuando es lo bastante grave, lesiona también a los riñones.

La solución consiste en reducir la presión arterial por medio de medicamentos o cambios de los hábitos.11

Principales causas de discapacidad por enfermedades no transmisibles 
En hombres mayores

- Trastornos respiratorios (sobre todo enfermedad pulmonar obstructiva crónica),

- Enfermedades cardiovasculares (sobre todo cardiopatía isquémica y accidente cerebrovascular)

- Cuadros neuropsiquiátricos (sobre todo demencia).

En mujeres mayores

- Cuadros neuropsiquiátricos (sobre todo demencia y también depresión grave),

- Trastornos respiratorios (sobre todo enfermedad pulmonar obstructiva crónica),

- Enfermedades cardiovasculares (accidente cerebrovascular y cardiopatía isquémica)

- Pérdida de visión por cataratas y glaucoma.

Las neoplasias malignas son una causa importante de muerte en los hombres y mujeres mayores, pero su contribución al número de años de vida con discapacidad es menor que la de los cuadros citados. La obesidad sobrecarga al corazón y a los pulmones. Eleva la presión arterial y se asocia a un ascenso de las lipoproteínas de baja densidad ("colesterol malo"); favorece el desarrollo de la diabetes mellitas de aparición adulta. También incrementa el riesgo de cáncer de colon, riñón y endometrio. Además, al poner un peso excesivo en las piernas, agrava laartritis de sus articulaciones.

La solución: perder peso y mantener un peso saludable; para esto, resulta útil hacer ejercicio y reducir la ingesta calórica.12

Acción comunitaria para ciudadanos de la tercera edad

Casi todas las medidas preventivas y de detección recomendadas en la sección anterior se describen desde una perspectiva clínica. Por fortuna, muchas de las necesidades de las personas mayores pueden satisfacerse parcialmente con actividades a nivel comunitario. Los organismos de la comunidad, los programas de extensión hospitalarios, las iglesias, los grupos de jubilados, los centros de la tercera edad y los vecindarios pueden organizar programas de educación para la salud, pruebas sencillas de detección y, en caso necesario, remitir a los implicados a los lugares adecuados.

Cuando se trata de cambiar los hábitos, el enfoque de grupo tiene una gran ventaja: cuando personas que comparten necesidades similares trabajan dentro de un grupo, pueden enseñarse y motivarse mutuamente con más eficacia, aguzar mejor sus capacidades mediante la 
imitación y la repetición y recompensarse unas a otras por mantener un estilo de vida más sano. Un grupo amistoso consigue estos objetivos de una forma mucho más sutil, global y potente que cualquier médico, enfermera, maestro o experto, porque muchos ancianos perciben a estos extraños como diferentes de ellos.

Con frecuencia, los grupos de autoayuda surgen por iniciativa de un profesional de la salud y la responsabilidad de su dirección recae poco a poco en líderes del grupo. La OMS reconoce que el movimiento de autoayuda en todas sus formas es un vehículo importante para conseguir la meta de "salud para todos". Unos grupos pueden centrarse en el ejercicio regular: por ejemplo, un club de pérdida de peso puede organizar paseos diarios de un kilómetro. Los ancianos que han perdido a un ser querido pueden encontrar a otros que están pasando por los mismos quebrantos para discutir los aspectos prácticos y emocionales de la adaptación a la nueva situación. El grupo ayuda a los ancianos dolientes a modificar su enfoque en el pasado, a empezar a planificar la "vida de ahora en adelante" y a valerse por sí mismos en sus nuevas circunstancias. Compartir estas experiencias puede aliviar la depresión ciertamente no la empeora. Hablando, caminando juntos o compartiendo pasatiempos activos, los miembros del grupo ayudan a combatir la depresión con medios fisiológicos.

La frecuencia de las pérdidas sociales y de los episodios de depresión aumenta progresivamente en los últimos años de la vida. El antídoto natural es la participación en grupos que comparten intereses o aficiones, actividades y comidas o meriendas, que proporcionan un "cambio de escenario" a los que viven solos y proveen una red de apoyo social. El apoyo se da y se recibe al mismo tiempo; los que dan y los que reciben cambian de puesto a medida que lo hacen las circunstancias. Con gran frecuencia, dar es mucho más útil que recibir.

Las claves de un buen envejecimiento son:

- Mantener activo el cuerpo,

- Mantener activo el cerebro y

- Mantener y ampliar las relaciones sociales.

Para promover la salud de los ancianos, la comunidad también puede:

- Proporcionar, o incitar a otros a proporcionar, lugares donde los ancianos puedan reunirse y compartir actividades.

- Proporcionar periódicamente medidas sencillas de promoción de la salud y detección de las enfermedades en los lugares donde los ancianos se reúnen. 
- Facilitar el acceso de los ancianos o discapacitados a los negocios y edificios públicos,

- Adoptar y poner en práctica medidas de salud, seguridad y calidad de vida en los centros residenciales.

- Conseguir la cooperación de organizaciones, escuelas, medios de comunicación, iglesias y otros transmisores de valores para hacer que la vida de la comunidad sea "más fácil para los viejos".13

\section{Actividades físicas}

1. Quiero ser joven: esta actividad es el desarrollo de los ancianos con ejercicios que ellos quieran ser sin tener una voz de mando.

2. Gimnasia matutina: esta actividad se hace cuando él se levanta en su cubículo ante de desayunar.

3. Conversatorio sobre los ejercicios en las diferentes enfermedades que ellos tienen: esta actividad le darán a ellos un panorama para poder tomar menos medicamentos para su salud.

4. A jugar en la tercera edad: esta actividad le dará a ellos unión teniendo diferente carácter y buscando el valor colectivo.

5. Jugando con la bola del mundo: esta actividad se hace en un círculo y se pasan una pelota y se le hace pregunta de diferentes acontecimientos del mundo.

Todas estas actividades se hacen todos los días de la semana de lunes a viernes, sábado y domingo las actividades que son para el cubículo y el hogar.14

\section{El adulto mayor en Cuba}

Especial significación tiene esta fecha en la actualidad, en que el mundo vive un proceso acelerado de envejecimiento. Cuba que no escapa a este fenómeno mundial y posee una esperanza de vida al nacer de casi 80 años, concede un cuidado prioritario a este segmento poblacional, que suma casi 16 por ciento del total de habitantes de la Isla. Actualmente, el Adulto mayor en Cuba se ha integrado al exitoso programa de la Universalización de la enseñanza y en su totalidad egresan de las aulas con el nivel superior. En estos espacios educacionales se ofrecen asignaturas que le aportan al anciano una Cultura general integral. Muchos saben manejar las Nuevas tecnologías y hacen uso de interesante Software Los crecientes desafíos que nos impone el desarrollo tecnológico requieren, cada vez más, de la formación de una cultura informática. En estos años han sido tan vertiginosos los cambios 
ocurridos en el mundo, que resulta necesario conformar los sistemas educacionales con un carácter abierto, a fin de facilitar la adaptación de los mismos a las condiciones existentes. Somos del criterio que laRevolución ha puesto equipos en nuestras manos, con el fin de desarrollar una cultura integral en los estudiantes, así como su actividad cognoscitiva esté vinculada con todos los aspectos de la vida y de la personalidad humana. En la medida en que el hombre descubre esa riqueza de conocimientos, surge en él la necesidad de conocer, de saber, por lo que se forma un pensamiento dialéctico e independiente. LaInformática, unida a las comunicaciones, posibilita prácticamente a todo el mundo, y es uno de los temas más atrayentes en la Universidad del Adulto Mayor de la 3era edad, lo que demuestra que nunca es tarde para adquirir conocimientos y darle un vuelco a la vida. Vivencias inigualables elevaron la autoestima del Adulto Mayor de la 3era edad al comprobar la utilidad de los diferentes cursos, que dan la posibilidad de ser útil en una etapa de la vida que experimenta cambios físicos y mentales o el acceso inmediato a la información.15

\section{Objetivos}

- Mejorar sus vidas

- Permitir una digna vejez y una adecuada reinserción familiar y comunitaria

- Permitir a los matriculados un mayor crecimiento como seres humanos

- Realizar sus proyectos de vida.16

\section{Diabetes Mellitus}

Proviene del Latín diabetes. Como término para referirse a la enfermedad caracterizada por la eliminación de grandes cantidades de orina ("poliuria"), empieza a usarse en el siglo I en el sentido etimológico de paso, aludiendo al paso de orina de la poliuria. Fue acuñado por el filósofo griego Arateus de Cappadocia. La palabra Mellitus (griego mel, miel) se agregó en 1675 por Thomas Willis cuando notó que la orina de un paciente diabético tenía sabor dulce.

\section{Características generales}

La Diabetes mellitus (DM) es hoy día un problema creciente de salud tanto para el mundo desarrollado como subdesarrollado. En 1994 existían 100 millones de personas con Diabetes, 165 millones en el año 2000.

Las personas con Diabetes tienen una esperanza de vida reducida y una mortalidad dos veces mayor que la población general. Una mejoría en el cuidado de la diabetes aumentaría la esperanza de vida de estas personas, pero esto a su vez llevaría una mayor incidencia de complicaciones micro vasculares (Nefropatía y Retinopatía) y macro vasculares (Enfermedades coronarias, cerebro vasculares y vasculares periféricas ya que la edad y la 
duración de la Diabetes son los principales factores de riesgo no controlables, por lo que será necesario aplicar los conocimientos existentes o desarrollar tecnologías capaces de prevenir la aparición de la enfermedad y de sus complicaciones, lo que contribuirá a reducir la carga económica que origina en la sociedad, que se concentra sobre todo en los gastos de hospitalización provocados por las complicaciones.

Por estas razones, la 42 Asamblea Mundial de la Salud del 29 de mayo de 1989, emitió la Resolución WAH 42.36, en la que se insta a los Estados Miembros a determinar la importancia nacional de la Diabetes, aplicar medidas poblacionales locales para prevenir y controlarla, promover colaboraciones interestatales para el adiestramiento y educación continuada sobre las acciones cotidianas que conlleva el tratamiento de las personas con Diabetes, así como las acciones de salud pública, estableciendo un enfoque integrado a nivel de la comunidad.

La Organización Mundial de la Salud reconoce tres formas de diabetes mellitus: tipo 1, tipo 2 y diabetes gestacional (ocurre durante el embarazo), 3 cada una con diferentes causas y con distinta incidencia. Varios procesos patológicos están involucrados en el desarrollo de la diabetes, le confieren un carácter autoinmune, característico de la DM tipo 1, hereditario y resistencia del cuerpo a la acción de la insulina, como ocurre en la DM tipo 2. Para el 2000, se estimó que alrededor de 171 millones de personas eran diabéticos en el mundo y que llegarán a 370 millones en 2030.

Este padecimiento causa diversas complicaciones, dañando frecuentemente a ojos, riñones, nervios y vasos sanguíneos. Sus complicaciones agudas son (hipoglucemia, cetoacidosis, coma hiperosmolar no cetósico) consecuencia de un control inadecuado de la enfermedad mientras sus complicaciones crónicas (cardiovasculares, nefropatías, retinopatías, neuropatías y daños microvasculares) son consecuencia del progreso de la enfermedad. El Día Mundial de la Diabetes se conmemora el 14 de noviembre.

Actualmente se piensa que los factores más importantes en la aparición de una diabetes tipo 2 son, además de una posible resistencia a la insulina e, el exceso de peso y la falta de ejercicio. De hecho, la obesidad abdominal se asocia con elevados niveles de ácidos grasos libres, los que podrían participar en la insulino-resistencia y en el daño a la célula betapancreática. Para la diabetes tipo 1 priman, fundamentalmente, la herencia genética, o bien, alguna patología que influya en el funcionamiento del páncreas (diabetes tipo 1 fulminante).

La actividad física mejora la administración de las reservas de azúcares del cuerpo y actúa de reguladora de las glucemias. Las reservas de aumentan y se dosifican mejor cuando el cuerpo está en forma, ya se queman con más facilidad, reservando más los hidratos de carbono para esfuerzo intensos o en caso de que la actividad sea muy larga que las reservas aguanten más tiempo. En realidad las causas de Diabetes todo tipo2 son mayoritariamente desconocidas, y por ende, no existe ninguna estrategia de prevención.17 


\section{Diagnóstico}

Se basa en la medición única o continua (hasta 2 veces) de la concentración de glucosa en plasma. La Organización Mundial de la Salud (OMS) estableció los siguientes criterios en 1999 para establecer con precisión el diagnóstico:

- Síntomas clásicos de la enfermedad (Poliuria, Polidipsia, Polifagia y pérdida de peso inexplicable) más una toma sanguínea casual o al azar con cifras mayores o iguales de $200 \mathrm{mg} / \mathrm{dl}(11.1 \mathrm{mmol} / \mathrm{L})$.

- Medición de glucosa en plasma en ayunas mayor o igual a $126 \mathrm{mg} / \mathrm{dl}$ (7.0mmol/L). Ayuno se define como no haber ingerido alimentos en al menos 8 horas.

- La prueba de tolerancia a la glucosa oral (curva de tolerancia a la glucosa). La medición en plasma se hace dos horas posteriores a la ingesta de $75 \mathrm{~g}$ de glucosa en $30 \mathrm{ml}$ de agua; la prueba es positiva con cifras mayores o iguales a $200 \mathrm{mg} / \mathrm{dl}$.

\section{Tipos de Diabetes}

1. Diabetes mellitus tipo 1 , diabetes mediada por procesos autoinmunes diabetes idiopática.

2. Diabetes mellitus tipo 2 .

3. Alteración del metabolismo de la glucosa.

4. Diabetes gestacional.18

\section{Síntomas y signos de la diabetes mellitus no tratada}

Los síntomas principales de la diabetes mellitus son emisión excesiva de orina (poliuria), aumento anormal de la necesidad de comer (polifagia), incremento de la sed (polidipsia), y pérdida de peso sin razón aparente.[6] En ocasiones se toma como referencia estos tres síntomas (poliuria, polifagia y polidipsia o regla de las $3 \mathrm{P}$ ) para poder sospechar diabetes tipo 2 ya que en su mayoría son los más comunes en la población. La Organización Mundial de la Salud reconoce tres formas de diabetes mellitus: tipo 1, tipo 2 y diabetes gestacional (ocurre durante el embarazo), cada una con diferentes causas y con distinta incidencia.

En el caso de que todavía no se haya diagnosticado la DM ni comenzado su tratamiento, o que no esté bien tratada, se pueden encontrar los siguientes signos (derivados de un exceso de glucosa en sangre, ya sea de forma puntual o continua):

- Poliuria, polidipsia y polifagia. 
- Pérdida de peso a pesar de la polifagia.

- Fatiga o cansancio.

- Cambios en la agudeza visual.19

\section{Estilo de Vida}

La importancia de un plan de alimentación en el manejo de la diabetes: Los niveles del azúcar en la sangre pueden ser controlados, hasta cierto punto, por medio de una dieta apropiada, ejercicio y el mantener un peso saludable. Un estilo de vida saludable también puede ayudarle a controlar o bajar su presiónsanguínea y a controlar las grasas en la sangre, reduciendo el riesgo de la cardiopatía.

Un plan de alimentación apropiado debería incluir la repartición de comidas pequeñas a través del día para mantener los niveles del azúcar en la sangre estables. Consumir una comida grande solo una o dos veces al día puede causar niveles extremos de azúcar altos o bajos. Además, si el régimen de ejercicio se cambia, deben hacerse los cambios correspondientes en la dieta, para mantener el control del peso y para controlar los niveles del azúcar en la sangre. Tenga o no tenga diabetes, el seguir las guías de la pirámide de los alimentos creada por el Departamento de Agricultura de Estados Unidos (US Department of Agriculture), para una alimentación apropiada es beneficioso para su salud. La pirámide de los alimentos está construida como una pirámide para demostrar una base de granos, pan, cereal, arroz y pasta (los más necesitados por nuestro cuerpo) con las grasas, los aceites y los dulces en la punta de la pirámide, demostrando que nuestros cuerpos solo necesitan una cantidad limitada de éstos.

Los Almidones proveen energía para el cuerpo, vitaminas, minerales y fibra. Aun cuando están llenos de carbohidratos que elevan los niveles de azúcar rápidamente, los almidones son esenciales para una dieta saludable. Ejemplos de almidones incluyen los siguientes:

- El pan.

- Los granos.

- La pasta.

- $\quad$ El Cereal.

- Ciertos vegetales.20

\section{Tratamiento}




\section{Tratamiento no farmacológico}

Este incluye lo siguiente:

- Educación: es de vital importancia educar al paciente diabético e involucrar al núcleo familiar en esta actividad. Para ello deben usarse mensajes sencillos, acordes con la edad y el nivel escolar del paciente.

- Dieta: no debe ser hipercalórica, pero al mismo tiempo debe evitarse la malnutrición iatrogénica. Debe ser rica en vitaminas y minerales (insistir en la ingesta de agua, calcio, potasio y vitamina B 12), y la distribución calórica será igual que en los adultos jóvenes. En los pacientes obesos, lograr una pérdida de peso del $10 \%$ debe ser considerado positivo y suficiente.

- Ejercicio físico: la actividad física es muy productiva para el paciente diabético, ya que mejora la sensibilidad a la insulina. Se recomienda la caminata en el horario de la mañana durante $1 \mathrm{~h}$ y como mínimo 4 veces a la semana, siempre que no existan contraindicaciones para su realización.

La fuerte asociación de la Diabetes Mellitus (DM) con la obesidad, sugiere que los primeros esfuerzos terapéuticos deben encaminarse a tratar de mantener un peso adecuado. Los sujetos con sobrepeso u obesidad, independientemente de que sus valores de glucosa sean normales, deben hacer dieta e incrementar la actividad física, por lo que siempre son beneficiosas las intervenciones a nivel de la comunidad que estén encaminadas a la consecución de dichos objetivos terapéuticos. Ensayos clínicos como el DPP, el DREAM y otros, evidencian que una intervención intensiva sobre el estilo de vida, proporciona la mayor reducción de los factores de riesgo cardiovascular y tiene un favorable perfil de seguridad, carece de efectos indeseables serios, y aporta otros beneficios a la salud.

\section{Tratamiento farmacológico}

Debe considerarse su empleo en el paciente cuando con la dieta y el ejercicio físico no se consiga un adecuado control de la DM, tras un período razonable (4-6 semanas) después del diagnóstico.

\section{Antidiabéticos orales}

Para su administración es necesario precisar si predomina la elevación de la glucemia posprandial o en ayuna. Si está elevada la primera, se utilizarán de preferencia los inhibidores de la alfaglucosidasa, los secretagogos de acción rápida, o los medicamentos que incrementen la sensibilidad a la insulina. Por el contrario, cuando la glucemia basal está elevada, lo más conveniente es el empleo de los secretagogos clásicos y también de los sensibilizadores de la insulina. De estos medicamentos, los más usados son: 
- Sulfonilureas: las sulfonilureas constituyen excelentes fármacos de primera línea para el tratamiento de la DM, cuya acción fundamental es estimular la secreción pancreática de insulina. En los ancianos se deben evitar las de acción prolongada y de gran potencia por el riesgo de hipoglucemia. La mayoría de los autores recomiendan la gliclasida, a la dosis de 80 a 320 mg diarios, así como también la glimeperida (1 a $8 \mathrm{mg} /$ día) que, aunque es de acción larga, produce poca hipoglucemia. La gliquidona (15 a $90 \mathrm{mg} /$ día) y la tolbutamida (500 mg a 2 g/día) también son opciones adecuadas para el tratamiento del paciente con DM, mientras que, por el contrario, la glibenclamida es muy potente, y por tanto, su uso conlleva un alto riesgo de hipoglucemia. Se aconseja comenzar con dosis bajas del medicamento a usar, e ir realizando incrementos progresivos basándose en los controles de glucemia. Se debe tener precaución con este grupo de medicamentos en la insuficiencia renal y hepática.

- Biguanidas: la única biguanida recomendada para su uso es la metformina, a la dosis de $500 \mathrm{mg}$, a $2 \mathrm{~g}$ diarios. Este medicamento no produce hipoglucemia y es el ideal para el paciente obeso no controlado aceptablemente solo con dieta y ejercicio físico. Está contraindicada en presencia de insuficiencia renal, cardíaca y hepática, enfermedades que cursen con hipoxia crónica, alcoholismo, o mala calidad de vida.

- Inhibidores de la alfaglucosidasa: la acarbosa, a la dosis de 50 a $100 \mathrm{mg}$ diarios divididos en 2 ó 3 tomas, se debe administrar de preferencia antes de las principales comidas. Este medicamento puede ser útil para controlar la hiperglucemia posprandial. No produce hipoglucemia, por lo que su uso da alguna seguridad, sin embargo, pueden tener efectos adversos, tales como, diarrea y flatulencia, por lo que en ocasiones no es bien aceptado por los pacientes.

- Tiazolidinedionas (TZD): las TZD más usadas son la troglitazona, la pioglitazona y la rosiglitazona, que mejoran la sensibilidad a la insulina, sobre todo, a nivel del músculo sin incrementar el peso corporal, y no producen hipoglucemia. Tienen un efecto beneficioso sobre los lípidos y la tensión arterial, y los estudios a largo plazo evidencian que pueden evitar la disfunción de la célula beta. Se acepta su empleo en el caso de la presencia una disfunción renal, por lo que hasta hace algunos años constituían los antidiabéticos orales de elección en los ancianos; sin embargo, recientemente se ha asociado la falla cardíaca congestiva con el uso de estos medicamentos. En un estudio en el cual se utilizaron TZD en pacientes con disfunción sistólica, no se hallaron diferencias entre las causas de mortalidad, pero sí se elevó la necesidad de hospitalización en el grupo de estudio $(4,5 \mathrm{mg} /$ día de rosiglitazona vs. 3,5 mg/día de placebo), empeoró el cuadro clínico cardiovascular, y, aunque este resultado no fue estadísticamente significativo, sería razonable tener precaución. Se precisó, además, que este efecto no depende de la dosis empleada ni del tiempo de 
exposición al medicamento, y sí del aumento del volumen plasmático, por lo que, considerando que los ancianos tienen una sensibilidad mayor al aumento de volumen, se debe tener precaución con el empleo de las TZD en estos pacientes con enfermedad cardíaca significativa y/o HTA.Los resultados del estudio DREAM, indicaron que la rosiglitazona fue tan efectiva en demorar o prevenir la DM como la modificación del estilo de vida, en los estudios Finlandés y DPP; sin embargo, este es un medicamento caro, y su uso se asocia con un incremento de 7 veces de la frecuencia de aparición de insuficiencia cardíaca congestiva.

La terapia combinada de fármacos orales puede ser una alternativa útil para retrasar la insulinización de algunos pacientes en la medida de lo posible.

\section{Insulina:}

Además de en los casos excepcionales, pero posibles, como son los pacientes con una DM 1, puede ser necesaria en algunos pacientes con DM 2 por diversas circunstancias:

- Contraindicaciones para el uso de hipoglucemiantes orales (insuficiencia renal o hepática grave, trastornos digestivos).

- Presencia de procesos intercurrentes que provocan descompensación de la glucemia, pudiendo ser necesaria la insulinización temporal.

- Fracaso del tratamiento con dieta-ejercicio e hipoglucemiantes orales, por no consecución de los objetivos de control pactados con el paciente.

- Descompensaciones hiperglucémicas agudas.

En ocasiones, la situación personal o familiar del paciente dificulta el tratamiento insulínico, por lo que ante estas condiciones, puede ser de utilidad la combinación de algún hipoglucemiante oral con una sola dosis de insulina intermedia en la noche. Generalmente, la insulina es mal aceptada por el paciente, y hay casos en los que las dificultades, en relación con su administración, son evidentes, por tanto, debemos tratar de implicar a los familiares o los cuidadores habituales del anciano, pues su colaboración no solo es recomendable, sino que puede ser esencial.

\section{El heberprot-p:}

Heberprot-P es un medicamento novedoso y único prescrito para la terapia de la úlcera del pie diabético (UPD) basado en el factor de crecimiento humano recombinante (FCHrec.), mediante infiltración intralesional directamente en el sitio de la herida. La terapia con HEBERPROT-P llena el espacio de una necesidad médica no satisfecha para el tratamiento de las úlceras complejas del pie diabético. Es una formulación inyectable que, administrada 
a través de infiltración intralesional, constituye un tratamiento adyuvante para acelerar la cicatrización de úlceras profundas, complejas, derivadas de este desorden metabólico, tanto neuropáticas como neuroisquémicas, con índice de presión tobillo/brazo (T/B) entre 0,3-0,8. El HEBERPROT-P ha sido aplicado con éxito en más de 800 pacientes cubanos aquejados de UPD y en este momento se trabaja aceleradamente en la extensión, a través de la Dirección Nacional de Atención Primaria de Salud, y de conjunto con la Dirección Nacional de Postgrado y el Grupo Nacional de pesquisa activa del MINSAP en implementar el uso del HEBERPROT-P® y el manejo integral de la UPD en todos los servicios de atención al paciente diabético en los 498 policlínicos de Cuba.21

\section{Tratamiento de la cormobilidad}

Por último, es imprescindible tener en cuenta para garantizar una atención integral a estas personas, las otras enfermedades que presentan estos pacientes con mucha frecuencia debido a su edad avanzada, y que están, además, en relación con la DM, tales como, HTA, cardiopatía isquémica, dislipidemia y estado procoagulante, entre otras, e indicar el tratamiento adecuado en cada caso. Finalmente, es necesario tener presente que:

- La DM es una enfermedad frecuente en los ancianos y su prevalencia aumenta con la edad.

- La mayoría de los ancianos tienen una alteración en la secreción de insulina y en la sensibilidad periférica a esta hormona, lo que favorece la aparición de la DM a esta edad.

- Los objetivos terapéuticos dependen de la situación funcional del enfermo.

- La educación, la dieta y el ejercicio físico son pilares básicos en el tratamiento no farmacológico.

- Las sulfonilureas de baja potencia y acción corta son las ideales para evitar las hipoglucemias.

- Las biguanidas, para mejorar la acción insulínica, y los inhibidores de la alfaglucodidasa, para el control de la hiperglucemia posprandial, pueden ser indicadas en el anciano con diabetes.

- Se debe utilizar insulina en circunstancias especiales.

- El tratamiento de la comorbilidad es de gran importancia en el tratamiento integral a estos pacientes. 22 


\section{Ejercicio físico}

El ejercicio es otro elemento muy importante en el tratamiento de la diabetes. Primero que todo, es importante porque en la mayoría de casos de diabetes, la persona debe bajar un poco de kilos y el ejercicio es muy importante en este procedimiento. El ejercicio también afecta los niveles de insulina que produce el cuerpo y sensibiliza los tejidos a la insulina.23

\section{Complicaciones de la diabetes:}

\section{Complicaciones}

Independiente del tipo de diabetes mellitus, un mal nivel de azúcar en la sangre conduce a las siguientes enfermedades. Bases son las modificaciones permanentes de las estructuras constructoras de proteínas y el efecto negativo de los procesos de reparación, ej.: la formación desordenada de nuevos vasos sanguíneos.

- Daño de los pequeños vasos sanguíneos (microangiopatía).

- Daño de los nervios periféricos (polineuropatía).

- Heridas difícilmente curables y la mala irrigación sanguínea de los pies, puede conducir a laceraciones y eventualmente a la amputación de las extremidades inferiores.

- Daño de la retina.

- Daño renal desde la nefropatía incipiente hasta la Insuficiencia renal crónica terminal

- Hígado graso o Hepatitis de Hígado graso.

- Daño de los vasos sanguíneos grandes (macroangiopatía): trastorno de las grandes Arterias.

- Esta enfermedad conduce a infartos, y trastornos de la circulación sanguínea en las piernas. En presencia simultánea de polineuropatía y a pesar de la circulación sanguínea crítica pueden no sentirse dolores: Debido a que el elevado nivel de glucosa ataca el corazón ocasionando daños y enfermedades coronarias: Sus primeras causas son la Diabetes avanzada, Hiperglucemia y el sobrepeso.

- Dermopatía diabética: o Daños a la piel.

- Hipertensión Arterial: Debido a la cardiopatía y problemas coronarios, consta que la hipertensión arterial y la diabetes son enfermedades "Hermanadas" 
La retinopatía diabética: es una complicación ocular de la diabetes, causada por el deterioro de los vasos sanguíneos que irrigan la retina del fondo del ojo. El daño de los vasos sanguíneos de la retina puede tener como resultado que estos sufran una fuga de fluido o sangre. Cuando la sangre o líquido que sale de los vasos lesiona o forma tejidos fibrosos en la retina, la imagen enviada al cerebro se hace borrosa.

Neuropatía diabética: neuropatía o trastorno neuropático a los cuales se asocian diabetes mellitus. Estos estados se piensan para resultar de lesiones microvascular diabética que involucra los vasos sanguíneos menores que suministra los nervios de los vasos. Los estados relativamente comunes que se pueden asociar a neuropatía diabética incluyen: Tercera parálisis del nervio; mononeuropatía; mononeuropatía múltiple; amiotrofia diabética; polineuropatía dolor; neuropatía autonómica; y neuropatía toracoabdominal

- La angiopatía diabética es una enfermedad de los vasos sanguíneos relacionada con el curso crónico de la diabetes mellitus, la principal causa de insuficiencia renal a nivel mundial. La angiopatía diabética se caracteriza por una proliferación del endotelio, acúmulo de glicoproteínas en la capa íntima y espesor de la membrana basal de los capilares y pequeños vasos sanguíneos. Ese espesamiento causa tal reducción de flujo sanguíneo, especialmente a las extremidades del individuo, que aparece la gangrena, que requiere amputación, por lo general de los dedos del pie o el pie mismo. Ocasionalmente se requiere la amputación del miembro entero. La angiopatía diabética es la principal causa de ceguera entre adultos no ancianos en los Estados Unidos. En Cuba, la tasa de angiopatías periféricas en la población diabética alcanza los 19,5 por cada 100 mil habitantes

- Cuando decimos que el Pie Diabético tiene una "base etiopatogénica neuropático" hacemos referencia a que la causa primaria que hace que se llegue a padecer un Pie Diabético está en el daño progresivo que la diabetes produce sobre los nervios, lo que se conoce como "Neuropatía". Los nervios están encargados de informar sobre los diferentes estímulos (nervios sensitivos) y de controlar a los músculos (nervios efectores). En los diabéticos, la afectación de los nervios hace que se pierda la sensibilidad, especialmente la sensibilidad dolorosa y térmica, y que los músculos se atrofien, favoreciendo la aparición de deformidades en el pie, ya que los músculos se insertan en los huesos, los movilizan y dan estabilidad a la estructura ósea.

El hecho de que una persona pierda la sensibilidad en el pie implica que, si se produce una herida, un roce excesivo, una hiperpresión de un punto determinado o una exposición excesiva a fuentes de calor o frío no se sientan. El dolor es, no lo olvidemos, un mecanismo defensivo del organismo que nos incita a tomar medidas que nos protejan de factores agresivos. Los diabéticos pueden sufrir heridas y no darse cuenta. Además, la pérdida de control muscular favorece como decimos la aparición de deformidades y éstas pueden al 
mismo tiempo favorecer roces, cambios en la distribución de los apoyos del pie durante la marcha y, en definitiva, predisponer a determinados puntos del pie a agresiones que, de no ser atajadas a tiempo, pueden resultar fatales.

\section{Complicaciones agudas:}

Estados hiperosmolares: comprenden dos entidades clínicas definidas: la (CAD) y el (CHNS). Ambos tienen en común -como su nombre lo dice, la elevación patológica de la sérica. Esto es resultado de niveles de glucosa sanguínea por encima de $250 \mathrm{mg} / \mathrm{dL}$, llegando a registrarse, en casos extremos más de $1000 \mathrm{mg} / \mathrm{dL}$. La elevada osmolaridad sanguínea provoca diuresis osmótica la cual pone en peligro la vida del paciente. La cetoacidosis suele evolucionar rápidamente, se presenta en pacientes con DM tipo 1 y presenta; en cambio el coma hiperosmolar evoluciona en cuestión de días, se presenta en ancianos con DM tipo 2 y no presenta. Tienen en común su gravedad, la presencia de deshidratación severa y alteraciones electrolíticas, el riesgo de coma, convulsiones, falla orgánica múltiple y muerte. Los factores que los desencadenan suelen ser: errores, omisiones o ausencia de tratamiento, infecciones agregadas -urinarias, respiratorias, gastrointestinales-, cambios de actividad física, cirugías, entre otros. Disminución del nivel de glucosa en sangre por debajo de los 50 $\mathrm{mg} / \mathrm{dL}$. Puede ser consecuencia de ejercicio físico no habitual o sobreesfuerzo, sobredosis de insulina, cambio en el lugar habitual de inyección, ingesta insuficiente de hidratos de carbono, diarreas o vómitos, etc. 24

\section{Seguimiento}

Además de un estudio médico adecuado, existen exámenes de laboratorio para monitorizar los órganos afectados en la diabetes mellitus (mediante control del nivel de glucosa, función renal, dislipidemia, etc.):

Pruebas de laboratorio de rutina de seguimiento y para monitorizar complicaciones en órganos blanco:

- Determinación de micro albuminuria en orina de 24 horas

- Hemoglobina glucosilada.

- Determinación de colesterol y trigliceridos en sangre.

- Creatininemia, uremia, electrolitos plasmáticos.

\section{Revisiones por especialistas que también ayudan a evitar complicaciones:}

- Revisión anual por oftalmología, preferentemente revisión del fondo de ojocon pupila dilatada. 
- Revisión del plan de alimentación por experto en nutrición.

- Revisión por podología por onicomicosis, tiña, uñas encarnadas (onicocriptosis)25

\section{Conclusiones.}

- Al concluir este trabajo pudimos profundizar nuestros conocimientos sobre el Adulto Mayor y una de las enfermedades más preocupantes de la actualidad: la Diabetes Mellitus, así como la importancia del ejercicio para combatirla.

\section{Recomendaciones.}

- Esperamos que con la siguiente revisión bibliográfica ayude a profesores de Cultura Física, personal de salud, estudiantes y otros que estén interesados en el tema.

\section{Referencias bibliográficas.}

Aplicación Web del Adulto Mayor, Joven Club de Computación.

Datos aportados por profesores de Cultura Física.

Ecurred: La tercera edad.

Lic. Cuello León, Eliester. El trabajo de la tercera edad en los establecimientos penitenciarios a través de la cultura física. 2011, Matanzas, Cuba

Díaz Otañez, J., Tendencias del Entrenamiento deportivo actual

Medicina Interna III; 4ta Edición.

Orosa Fraíz Teresa. (2003). La Tercera edad y la familia. Editorial Félix Varela. La Habana.

Keller Keller. Rosalia Orientación familiar y adulto mayor, Ciudad la Habana, mayo 2001.

Prieto R, O; Vega G, E. (1996). Temas de Gerontología. Ciudad de la Habana.

Caballero López A. et al. Terapia Intensiva. Cetoacidosis Diabética. pág. 4081.

Caídas en el anciano. Consideraciones generales y prevención Rev. Cubana Med Gen Integr 1999; 15(1):98-102http://bvs.sld.cu/revistas/mgi/vol15_1_99/mgi11199.htm

Emplenitud.com Caídas en la tercera http://www.enplenitud.com/nota.asp?notaid=1598

Borges Sosa Gladys J. Ponencia, Recordar es Vivir. Ponencia presentada en Edumayores 2008. Palacio de Convenciones 
Díaz, F. Y Becerra, F. Medición y evaluación de la Educación física y Deportes. Inversora Copy S.A., Caracas, 1981

Colectivo de Autores; Pedagogía. Editorial Pueblo y Educación. La Habana, 1984. Pp182301.

Hernández Ravelo Mariela. Trabajo de Diploma "Sitio Web para contribuir a la Divulgación de la Universidad del Adulto Mayor".

Programa nacional de atención al diabético

Red GEDAPS. Atención primaria y diabetes mellitus

Colectivo de Autores. Hospital Hermanos Ameijeiras. Organización Panamericana de la Salud.

Gallo Vallejo FJ. Manual del Residente de medicina familiar y comunitaria.El ejercicio físico.

Organización Mundial de la Salud. Manual de Diagnóstico y Tratamiento en Especialidades Clínicas. Cetoacidosis Diabética. 1ªEdición. pág. 172- 175. 2002.

Lovesio C. Cetoacidosis Diabética. Texto de Medicina Intensiva. $5^{\text {a }}$ Ed. 2000.pag. 1041-49. 3. MINSAP. Anuario Estadístico. Año 2004.

$\begin{array}{llll}\text { Saludalia.com Cristina } & \text { Castro } & \text { Cristóbal }\end{array}$ .Salud.http://www.saludalia.com/docs/Salud/web_saludalia/tu_salud/doc/diabetes/d oc/doc_caidas.htm

Wiki Pedia

Internet: Tratamiento de la Diabetes Mellitus

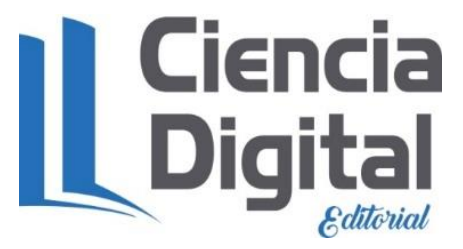




\section{PARA CITAR EL ARTÍCULO INDEXADO.}

Mederos González, A., \& González Pérez, E. (2019). El Adulto Mayor en la actualidad y la Diabetes Mellitus como una enfermedad preocupante. Anatomía Digital, 2(2), 61-83. https://doi.org/10.33262/anatomiadigital.v2i2.1081

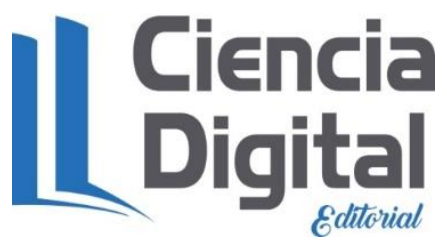

El artículo que se publica es de exclusiva responsabilidad de los autores y no necesariamente reflejan el pensamiento de la Revista Anatomía Digital.

El artículo queda en propiedad de la revista y, por tanto, su publicación parcial y/o total en otro medio tiene que ser autorizado por el director de la Revista Anatomía Digital.
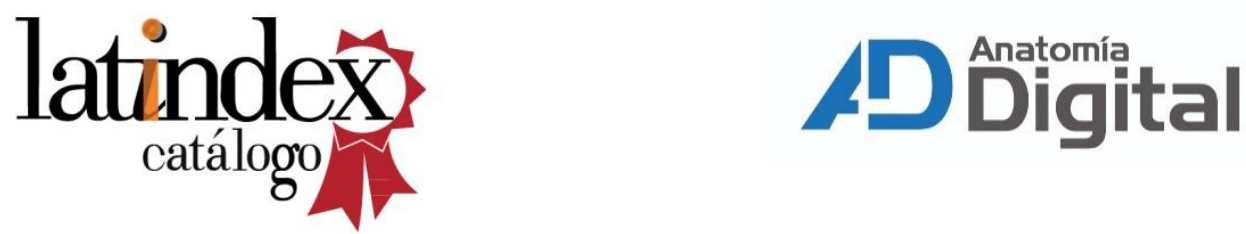